\title{
Efficient Topical Delivery of Chlorogenic Acid by an Oil-in-Water Microemulsion to Protect Skin against UV-Induced Damage
}

\author{
Shuji Kitagawa, * Kenta YoshiI, Shin-ya Morita, and Reiko Teraoka \\ Kobe Pharmaceutical University; 4-19-1 Motoyamakita-machi, Higashinada-ku, Kobe 658-8558, Japan.
} Received February 5, 2011; accepted March 10, 2011; published online March 15, 2011

\begin{abstract}
We examined the intradermal delivery of a hydrophilic polyphenol chlorogenic acid by in vitro study using excised guinea pig dorsal skin and Yucatan micropig skin. Skin accumulation as well as the solubility of chlorogenic acid in aqueous vehicles was much greater than for other polyphenols such as quercetin and genistein. However, since enhancement of skin delivery seemed to be necessary to exhibit its protective effects against oxidative damage of skin, we examined the effects of microemulsions as vehicles. Using microemulsions consisting of $150 \mathrm{~mm} \mathrm{NaCl}$ solution, isopropyl myristate, polyoxyethylene sorbitan monooleate (Tween 80) and ethanol, skin accumulation as well as solubility of chlorogenic acid further increased. Enhancement effect of an oil-in-water (o/w-type) microemulsion was greater than that of a water-in-oil (w/o-type) microemulsion possibly due to the greater increase in solubility. This finding was quite different from previous findings on relatively hydrophobic polyphenols such as quercetin and genistein. Pretreatment of guinea pig dorsal skin with chlorogenic acid containing microemulsion gel prevented erythema formation induced by UV irradiation. These findings indicate the potential use of hydrophilic chlorogenic acid with o/w-type microemulsion as a vehicle to protect skin against $\mathrm{UV}$ induced oxidative damage.
\end{abstract}

Key words chlorogenic acid; skin; intradermal delivery; microemulsion; UV irradiation

Chlorogenic acid (Fig. 1), which is a well-known antioxidant, is an abundant ingredient in coffee and is also found in many foods, such as sweet potatoes and apples. ${ }^{1-3)}$ It has been suggested that chlorogenic acid has anti-inflammatory and anticancer activities and is also effective to prevent cardiovascular disease ${ }^{4-6)}$ and to decrease blood glucose levels. ${ }^{7)}$ Like other plant polyphenols, its application for topical purposes, such as photoprotection against UV-induced skin damage (photoaging), skin cancer prevention, and skin care, is expected. ${ }^{8,9)}$ Different from other plant polyphenols, such as resveratrol, quercetin, and genistein, chlorogenic acid is hydrophilic and soluble in water. For hydrophilic compounds, lipid lamella in the stratum corneum works as a barrier to their intradermal and transdermal delivery ${ }^{10)}$; therefore, to apply chlorogenic acid for topical purposes, it is necessary to improve interadermal delivery.

Microemulsions consist of an aqueous phase, an organic phase, a surfactant and a co-surfactant component, which are thermodynamically stable and have been shown to have high solubilization capacity and to facilitate the skin permeation of both lipophilic and hydrophilic drugs. ${ }^{11,12}$ In previous studies we have clarified that microemulsions, especially a water-in-oil (w/o type) microemulsion consisting of $150 \mathrm{~mm}$ $\mathrm{NaCl}$ solution, isopropyl myristate, polyoxyethylene sorbitan monooleate (Tween 80) and ethanol markedly increased the intradermal delivery as well as solubility of hydrophobic polyphenols quercetin and genistein, whose solubility in both

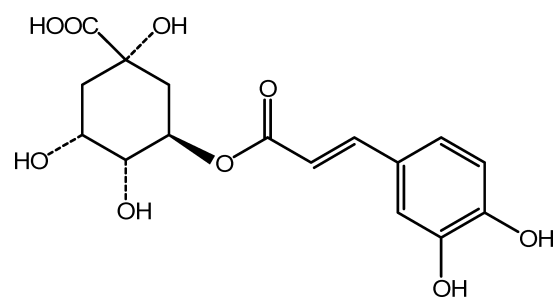

Fig. 1. Chemical Structure of Chlorogenic Acid aqueous and organic media is very low. ${ }^{13,14)}$ Although improvement of solubility does not usually lead to increased solute skin delivery, microemulsions effectively improved skin delivery of these polyphenols; therefore, microemulsions seemed to be useful as a vehicle for the skin delivery of solutes whose solubility in ordinal vehicles is limited. Different from quercetin and genistein, since chlorogenic acid is hydrophilic, moderate solubility in aqueous medium is expected, although for hydrophilic compounds permeation through stratum corneum lipid lamella is a big problem for their skin delivery. In this study we first examined the intradermal delivery of chlorogenic acid from aqueous medium. We also examined the effects of w/o-type and an oil-in water (o/w-type) microemulsions on its delivery. We furthermore examined the effects of the topical application of chlorogenic acid in vivo using guinea pigs on ultraviolet light-induced erythema formation in the presence and absence of microemulsion gel, in which co-surfactant ethanol concentration was minimized to avoid skin damage by alcohol.

\section{Results and Discussion}

We first examined the solubility of chlorogenic acid. As shown in Fig. 2, the solubility of chlorogenic acid in $150 \mathrm{~mm}$ $\mathrm{NaCl}$ solution was about $43 \mathrm{~mm}$, which was much greater than the values of other polyphenols revealed previously, such as quercetin $(0.31 \mathrm{~mm})^{13)}$ and genistein $(0.059 \mathrm{~mm}){ }^{14)}$ We examined the effects of three microemulsions using $150 \mathrm{~mm} \mathrm{NaCl}$ solution as an aqueous phase and isopropyl myristate as an oil phase, Tween 80 as a surfactant, and ethanol as a co-surfactant, whose compositions were listed in Table 1. Ethanol also works as an ingredient in the aqueous phase. Microemulsions, especially o/w-type microemulsions $\mathrm{A}$ and $\mathrm{K}$, further increased the solubility of chlorogenic acid seven or eight times compared with that in the control aqueous vehicle.

We next examined the topical delivery of chlorogenic acid. As shown in Fig. 3 for the results on excised guinea pig skin, 
the intradermal delivery of chlorogenic acid from a control aqueous vehicle $(1.49 \mu \mathrm{mol} / \mathrm{g}$ skin $)$ was much greater than those of quercetin $(0.034 \mu \mathrm{mol} / \mathrm{g} \text { skin })^{13)}$ and genistein $(0.10 \mu \mathrm{mol} / \mathrm{g}$ skin $) .{ }^{14)}$ One reason for the relatively high skin delivery of chlorogenic acid from the aqueous vehicle is pos-

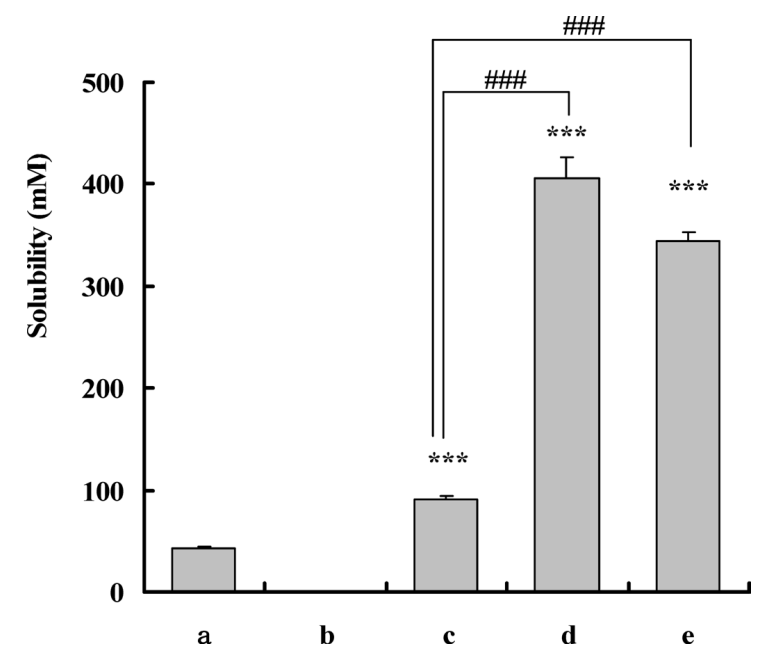

Fig. 2. Solubility of Chlorogenic Acid in $150 \mathrm{~mm} \mathrm{NaCl}$ Solution (a), Isopropyl Myristate (b), Microemulsion D (c), Microemulsion A (d), and Microemulsion $\mathrm{K}(\mathrm{e})$

Data are the means \pm S.D. of four experiments. $* * * p<0.001$, significantly different from the values in $\mathrm{NaCl}$ solution and isopropyl myristate. \# $p<0.001$, significant difference between data.

Table 1. Composition of Microemulsions Used in This Study

\begin{tabular}{ccccc}
\hline \hline Microemulsion & $\begin{array}{c}\text { Isopropyl } \\
\text { myristate }\end{array}$ & $\begin{array}{c}\mathrm{NaCl} \\
\text { solution }\end{array}$ & Tween 80 & Ethanol \\
\hline $\mathrm{A}$ & 8 & 25 & 20 & 47 \\
$\mathrm{~K}$ & 4 & 51 & 30 & 15 \\
$\mathrm{D}$ & 33 & 7 & 30 & 30 \\
\hline
\end{tabular}

Composition is given as $\%, \mathrm{w} / \mathrm{w}$.

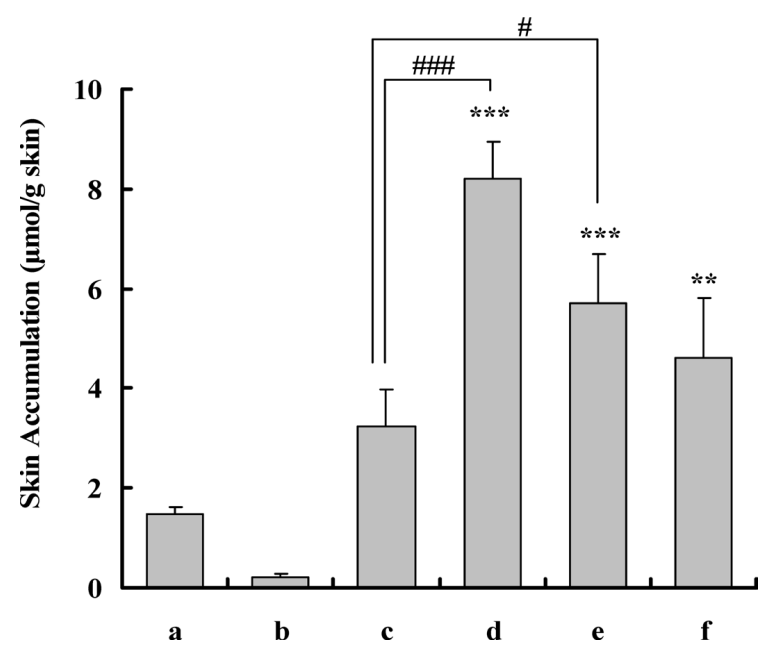

Fig. 3. Skin Accumulation of Chlorogenic Acid in Guinea Pig Skin Applied at Saturated Concentration in $150 \mathrm{~mm} \mathrm{NaCl}$ Solution (a), Isopropyl Myristate (b), Microemulsion D (c), Microemulsion A (d), Microemulsion $\mathrm{K}$ (e), and Microemulsion K Gel (f)

Data are the means \pm S.D. of four experiments. $* * p<0.01, * * * p<0.001$, significantly different from the values in $\mathrm{NaCl}$ solution and isopropyl myristate. $\# p<0.05$, $\# p<0.001$, siginificant difference between data. sibly the high solubility of chlorogenic acid. However, compared to the previous findings on skin accumulation of quercetin $(3.66 \pm 0.27 \mu \mathrm{mol} / \mathrm{g}$ skin) and genistein (5.92 \pm $1.48 \mu \mathrm{mol} / \mathrm{g}$ skin) by microemulsion $\left.\mathrm{D},{ }^{13,14}\right)$ skin accumulation of chlorogenic acid by aqueous vehicle was less and might be insufficient to exhibit its anti-oxidative effects in skin. Therefore, we next examined the effects of microemulsions using as vehicles. As shown in Fig. 3, skin accumulation further increased when microemulsions with saturated concentrations of chlorogenic acid were used, especially when o/w-type microemulsions $\mathrm{A}$ and $\mathrm{K}$ were used. The increase was 5.5 times and 3.8 times for microemulsion $\mathrm{A}$ and $\mathrm{K}$, respectively, compared with the accumulation from the aqueous vehicle. Since significant enhancement on skin delivery of chlorogenic acid was found for the o/w-type microemulsions, we continued the study by using microemulstion $\mathrm{K}$ to avoid the effect of a high concentration of ethanol on the skin. We next prepared microemulsion gel by adding $1.0 \%$ carbopol 981 to microemulsion $\mathrm{K}$ and examined its effect on the skin delivery of chlorogenic acid because gelation is necessary for in vivo application. The microemulsion $\mathrm{K}$ gel also increased skin accumulation, although it decreased to about $80 \%$ of skin accumulation without carbopol 981.

Relatively high skin accumulation of chlorogenic acid from the aqueous vehicle and further enhancement by o/wtype microemulsions were also confirmed when Yucatan micropig skin, which is hairless and has been suggested to show physiological similarity to human skin, ${ }^{15)}$ was used, as shown in Fig. 4.

To see the effects of topical application of chlorogenic acid on UV-induced skin damage, we observed the effects of chlorogenic acid added either with aqueous gel or microemulsion gel on UV-B-induced erythema formation using guinea pig. As shown in Fig. 5b, after UV-B irradiation, erythema formation was observed on the side of skin to which microemulsion $\mathrm{K}$ gel without chlorogenic acid was preloaded. On the other hand, on the side of skin to which $350 \mathrm{~mm}$ chlorogenic acid with the microemulsion gel was preloaded, erythema formation was significantly inhibited. Redness increase of skin measured with a color reader, which was expressed as the $a^{*}$ difference values on the skin surface

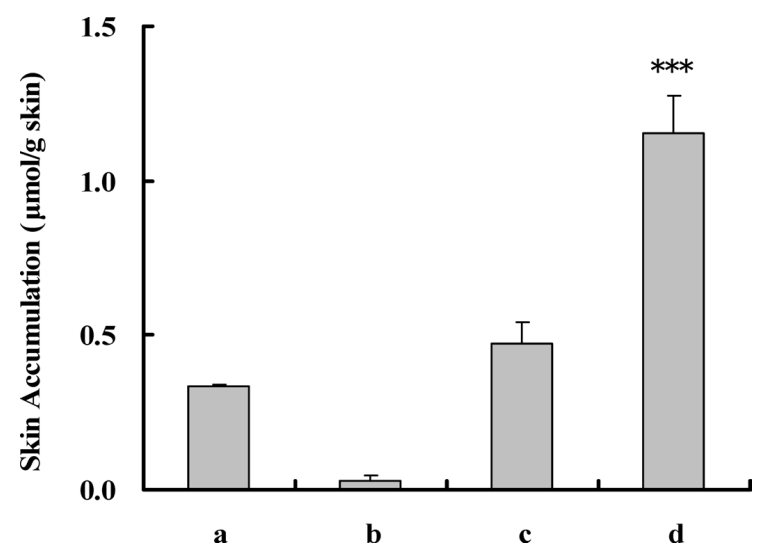

Fig. 4. Skin Accumulation of Chlorogenic Acid in Yucatan Micropig Skin Applied at Saturated Concentration in $150 \mathrm{~mm} \mathrm{NaCl}$ Solution (a), Isopropyl Myristate (b), Microemulsion D (c), and Microemulsion K (d)

Data are the means \pm S.D. of four experiments. $* * * p<0.001$, significantly different from the values in $\mathrm{NaCl}$ solution and IPM. 
(a)

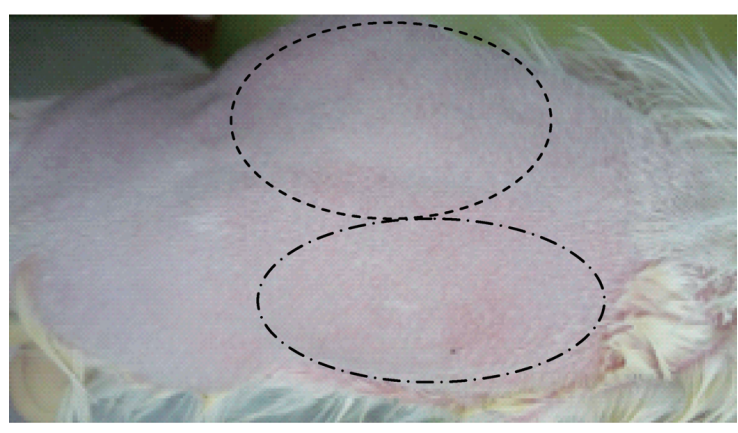

(b)

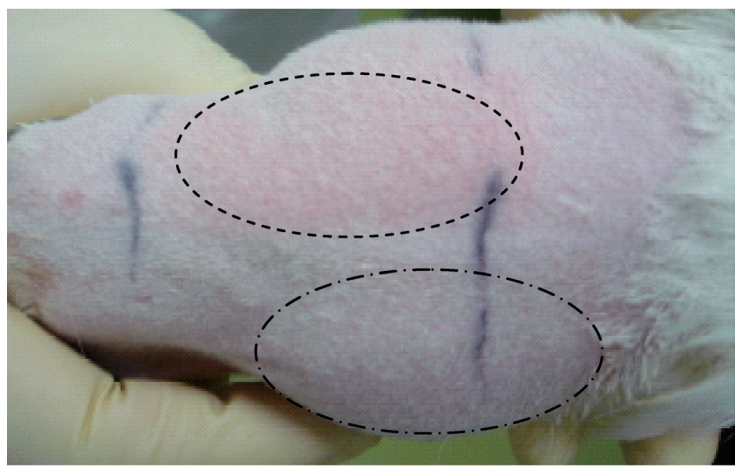

(c)

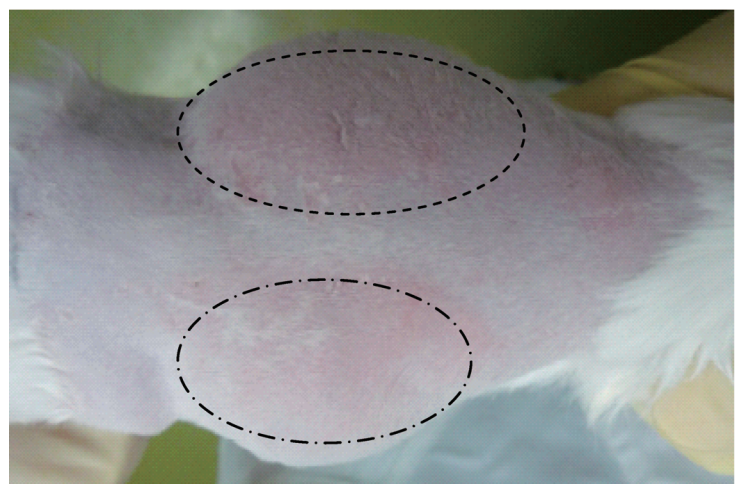

Fig. 5. Effect of Chlorogenic Acid on UV-B-Induced Erythema Formation before UV Irradiation (a) and after UV Irradiation (b and c)

Either Microemulsion K gel (b) or aqueous gel (c) without chlorogenic acid was added to the left side of skin (upper half in each picture indicated by the ellipse) and with $350 \mathrm{~mm}$ chlorogenic to the right side of skin (lower half in each picture indicated by the ellipse) at $20 \mathrm{~h}$ before UV irradiation.

before and after irradiation, was $5.0 \pm 0.8$ in control skin and $0.2 \pm 0.2$ in skin pretreated with chlorogenic acid containing microemulsion $\mathrm{K}$ gel $(n=6, p<0.001)$. On the other hand, preloading of $50 \mathrm{~mm}$ chlorogenic acid with aqueous gel did not inhibit erythema formation as shown in Fig. 5c (a* difference values were $2.9 \pm 0.6$ in control skin and $2.2 \pm 0.3$ in skin pretreated with chlorogenic acid, respectively $(n=3))$ possibly due to the insufficient concentration of chlorogenic acid in skin.

The findings obtained in this study revealed that microemulsions, especially o/w-type microemulsion, were effective for skin delivery of chlorogenic acid. This is quite dif- ferent from our previous findings on quercetin and genistein. ${ }^{13,14)}$ For these relatively hydrophobic polyphenols, w/otype microemulsion $\mathrm{D}$ was more effective than o/w-type microemulsion. Furthermore, using o/w-type microemulsion, chlorogenic acid showed marked skin accumulation compared with other polyphenols. ${ }^{13,14)}$ Skin accumulation of chlorogenic acid using microemulsion A $(8.21 \pm 0.73 \mu \mathrm{mol} / \mathrm{g}$ skin) or $\mathrm{K}(5.71 \pm 0.99 \mu \mathrm{mol} / \mathrm{g}$ skin) in a saturated condition was greater than that of quercetin $(3.66 \pm 0.27 \mu \mathrm{mol} / \mathrm{g} \text { skin })^{13)}$ and similar to or greater than that of genistein $(5.92 \pm 1.48$ $\mu \mathrm{mol} / \mathrm{g}$ skin $)^{14)}$ in a saturated condition using w/o-type microemulsion D.

The mechanism of the enhanced delivery of chlorogenic acid by $\mathrm{o} / \mathrm{w}$-type microemulsion is not clear. Incorporation ratio of chlorogenic acid into skin from total chlorogenic acid added in each vehicle at saturated condition was $0.31 \%$, $0.32 \%, 0.18 \%$ and $0.15 \%$, for aqueous vesicle, microemulsion $\mathrm{D}$, microemulsion $\mathrm{A}$ and microemulsion $\mathrm{K}$, respectively. Therefore, efficiency of incorporation rather slightly decreased in o/w-type microemulsions. From these findings contribution of the improvement of solubility seems to be large for the enhanced delivery of chlorogenic acid by o/wtype microemulsions. However, other factors should be involved. In the sink condition, the steady state flux of the diffusant, $m$, passing per unit area through the stratum corneum, which works as a barrier for the diffusant to penetrate the skin, is presented in Eq. 1, where $C_{\mathrm{D}}$ represents the donor solute concentration $\left(C_{\mathrm{D}}\right.$ equals solubility when saturated solute is added to the donor compartment); $D$, diffusion coefficient; $h$, thickness of stratum corneum; $K$, partition coefficient of solute between vehicle and skin shown in Eq. 2, and $C_{\mathrm{s}}$, solute concentration in stratum corneum. ${ }^{16)}$

$$
\begin{aligned}
& \frac{d m}{d t}=\frac{D K C_{\mathrm{D}}}{h} \\
& K=\frac{C_{\mathrm{s}}}{C_{\mathrm{D}}}
\end{aligned}
$$

The increased solubility of solute in the vesicle increases its thermodynamic stability in the vesicle, which leads to a decreased partition coefficient. As a result, increased solubility in the vesicle does not usually lead to increased intradermal drug delivery; therefore, microemulsions seem to work to increase either the diffusion coefficient or partition coefficient. Like other polyphenols, chlorogenic acid is expected to be mainly present in the interface region of the microemulsion. ${ }^{17)}$ Microemulsions, which reach the skin surface, seem to stimulate the partition of chlorogenic acid into the surface of the stratum corneum like carrier vesicles. Continuously and spontaneously fluctuating interfaces of microemulsions might enable high drug mobility and enhance the drug diffusion process in the stratum corneum. ${ }^{18)}$ Microemulsion may also enhance the skin delivery of chlorogenic acid, partly by mixing its component with lipid lamella of the stratum corneum. The larger enhancement effect of microemulsion A than microemulsion $\mathrm{K}$ is probably due to such effect of the high concentration of ethanol. ${ }^{19)}$

UV-B, which is an essential component of sunlight, crosses the epidermis and reaches the upper dermis, induces erythema formation, premature skin aging, inflammation and photocarcinogenesis, probably through the formation of reactive oxygen species. ${ }^{8,20)}$ It was demonstrated that photo-dam- 
age decreased when skin was supplemented with antioxidants. $^{21)}$ In this study, we revealed that the application of chlorogenic acid to skin prevented UV-B-induced erythma formation, as revealed for genistein in the previous study. ${ }^{14}$ ) This protective effect is possibly due to the reduction of reactive oxygen species by chlorogenic acid. The findings in this study indicate that application with o/w-type microemulsion as the vehicle is expected to enable efficient topical delivery of a hydrophilic polyphenols, chlorogenic acid, to protect skin against UV-induced oxidative damage.

\section{Experimental}

Materials Chlorogenic acid was purchased from Wako Pure Chemical Industries (Osaka, Japan). Tween 80 and isopropyl myristate were obtained from Nacalai Tesque (Kyoto, Japan). Ethanol and all other reagents were purchased from Wako Pure Chemical Industries. Carbopol 981 was provided by Nikko Chemicals (Tokyo, Japan). Dorsal skin was excised from guinea pigs following the protocol approved by the Animal Experimentation Committee of Kobe Pharmaceutical University. Pentobarbital sodium was used for anesthesia. Subcutaneous fat and other extraneous tissues were trimmed before using. Yucatan micropig skin (YMP skin set) was purchased from Charles River Japan (Yokohama, Japan). Fat and subdermal tissue were removed following the method of Fujii et al. ${ }^{22}$ )

Preparation of Microemulsion and Microemulsion Gel Microemulsions $\mathrm{A}, \mathrm{K}$ and $\mathrm{D}$ were prepared using $150 \mathrm{~mm} \mathrm{NaCl}$ solution as an aqueous phase and isopropyl myristate as an oil phase, Tween 80 as a surfactant, and ethanol as a co-surfactant at the composition shown in Table 1 by modifying the method of Lee et al. ${ }^{23}$ Each microemulsion was obtained by vortex mixing for a few minutes at $37^{\circ} \mathrm{C}$. Chlorogenic acid was added to the preformed microemulsions at saturated concentration as solubilized condition at $37^{\circ} \mathrm{C}$. The mean particle diameter of microemulsion $\mathrm{K}$ was $12.3 \pm 4.8 \mathrm{~nm}$, which was measured using a particle analyzer FPAR-1000 (Otsuka Electronics Co., Hiratsuka, Japan). Those of microemulsion A and D were as reported previously. ${ }^{13)}$ Microemulsion gel was prepared using carbopol 981 as a gelator. Carbopol 981 was added to $\mathrm{NaCl}$ solution with stirring and aqueous gel was prepared. Isopropyl myristate, Tween 80 and ethanol were mixed, added to this aqueous gel, and microemulsion gel was formed. Final concentration of carbopol 981 was $5.0 \%$ or $1.0 \%$, respectively, when $\mathrm{NaCl}$ solution itself or microemulsion $\mathrm{K}$ was used.

Measurement of Intradermal Delivery In vitro study on the skin incorporation of chlorogenic acid was examined as described previously. ${ }^{13,14}$ Guinea pig dorsal skin or Yucatan micropig skin was mounted in a Franz cell with a water jacket $\left(37^{\circ} \mathrm{C}\right)$. The available diffusion area was approximately $0.71 \mathrm{~cm}^{2}$ and the receptor cell had a capacity of about $4.7 \mathrm{ml}$. After $2 \mathrm{~h}$ pretreatment of the skin with $\mathrm{NaCl}$ solution and washing both donor and receptor compartments, $1 \mathrm{ml}$ vehicle containing chlorogenic acid was added to the donor compartment at saturated concentration under solubilized conditions, while phosphate-buffered saline (PBS) was added to the receptor compartment. Microemulsion gel was prepared as described above, and added similarly to the donor compartment to $1 \mathrm{ml}$. After $20 \mathrm{~h}$ treatment $(40 \mathrm{~h}$ treatment for Yucatan micropig skin), at which time the amount of chlorogenic acid penetrating the skin was close to the maximum level, the skin was removed from the cell, and the treated area was punched out and washed with ice-cold methanol. After drying at ambient temperature, the skin was weighed (about $0.09 \mathrm{~g}$ for guinea pig skin), minced, placed in $10 \mathrm{ml}$ methanol, and then homogenized using a tissue homogenizer Polytron PT3100 (Kinematica, Lucerne, Switzerland) at $5000 \mathrm{rpm}$ for $1 \mathrm{~min}$. The samples were then centrifuged and the supernatant layer was used to determine the concentration of chlorogenic acid by HPLC LC-10-AS (Shimadzu, Kyoto, Japan). Separation was achieved on a reversed-phase column (Mightysil RP-18 GP, $4.6 \mathrm{~mm}$ i.d., $150 \mathrm{~mm}$ ) using a mobile phase consisting of methanol, water and phosphoric acid $(60: 140: 1)$. SPD-10AVvp (Shimadzu) was used as the UV detector. The detection wavelength was $327 \mathrm{~nm}$. As an internal standard, trans-ferulic acid was used.

Measurement of Solubility Chlorogenic acid solubility was measured after incubation of an excess amount of chlorogenic acid in $150 \mathrm{~mm} \mathrm{NaCl}$ solution, isopropyl myristate or microemulsion at $37^{\circ} \mathrm{C}$ for about $20 \mathrm{~h}$. After quick centrifugation at $12000 \times \boldsymbol{g}$ for $1 \mathrm{~min}$, the concentration of the supernatant was determined by HPLC as described above.

Observation of Erythema Formation by UV Irradiation Erythema formation induced by UV irradiation was observed as described previ- ously, ${ }^{14)}$ following the protocol approved by the Animal Experimentation Committee of Kobe Pharmaceutical University. Fur on the dorsal skin of guinea pigs was cut using hair clippers and shavers and dirt on the skin was washed off. About $24 \mathrm{~h}$ later, microemulsion $\mathrm{K}$ gel containing chlorogenic acid was applied to the right side of the skin, while microemulsion $\mathrm{K}$ gel without chlorogenic acid was applied to the left side. After about $20 \mathrm{~h}$, microemulsion gel remaining on the skin surface was wiped off with wet gauze repeatedly. After drying the skin surface for $4 \mathrm{~h}$, both sides of the guinea pig dorsal skin were irradiated for $24 \mathrm{~min}$ by UV light at an intensity of $138 \mu \mathrm{W} / \mathrm{cm}^{2}$, measured with UV-Meter 340 (Ando Keiki Co., Tokyo, Japan). GL20SE UV-B lamps (Sankyo Denki Co., Tokyo), emitting a continuous light spectrum between 280 and $380 \mathrm{~nm}$ with a peak emission at $306 \mathrm{~nm}$, were used. Erythema formation was evaluated using photographs as well as skin redness measurement with the CR-13 color reader (Konica Minolta Holdings Inc., Tokyo, Japan).

Statistical Analysis Data were analyzed using the Kruskal-Wallis test. Individual differences between medians were examined using Dunn's multiple comparison test.

Acknowledgements This work was supported by a Grant from The Cosmetology Research Foundation, Tokyo, Japan. We thank Dr. Hitoshi Masaki and Dr. Yuri Okano, Nikkol Group Cosmos Technical Center Co., for instruction in the technique of the UV irradiation experiment.

\section{References}

1) Chinnici F., Bendini A., Gaiani A., Riponi C., J. Agric. Food Chem., 52, 4684-4689 (2004).

2) Tang Y.-Z., Liu Z.-Q., J. Agric. Food Chem., 56, 11025-11029 (2008).

3) Padda, M. S., Picha D. H., Int. J. Food Sci. Technol., 43, 1404-1409 (2008).

4) Jin U.-H., Lee J.-Y., Kang S.-K., Kim J.-K., Park W.-H., Kim J.-G., Moon S.-K., Kim C.-H., Life Sci., 77, 2760-2769 (2005).

5) Miceli N., Taviano M. F., J. Ethnopharmacol., 97, 261-266 (2005).

6) Bonita J. S., Mandarano M., Shuta D., Vinson V., Pharmcol. Res., 55, 187-198 (2007).

7) Bassoli B. K., Casolla P., Borba-Murad G. R., Constantin J., SalgueiroPagadigorria C. L., Bazotte R. B., Ferreira da Silva R. S. S., Medri de Souza H., Cell Biochem. Funct., 26, 320-328 (2008).

8) Caddeo C., Teskac K., Sinico C., Kristl J., Int. J. Pharm., 363, 183191 (2008)

9) Vayalil P. K., Elmets C. A., Katiyar S. K., Carcinogenesis, 24, 927936 (2003).

10) Kim J., Hwang J.-S., Cho Y.-K., Han Y., Jeon Y.-J., Yang K.-H., Skin Pharmacol. Appl. Skin Physiol., 14, 11-19 (2001).

11) Marti-Mestres G., Mestres J. P., Bres J., Martin S., Ramos J., Vian L., Int. J. Pharm., 331, 139-144 (2007).

12) Heuschkel S., Goebel A., Neubert R. H. H., J. Pharm. Sci., 97, 603631 (2008).

13) Kitagawa S., Tanaka Y., Tanaka M., Endo K., Yoshii A., J. Pharm. Pharmacol., 61, 855-860 (2009).

14) Kitagawa S., Inoue K., Teraoka R., Morita S., Chem. Pharm. Bull., 58, $398-401(2010)$.

15) Kurihara-Bergstrom T., Woodworth M., Feisullin S., Beall P., Lab. Animal Sci., 36, 396-399 (1986).

16) Barry B. W., "Percutaneous Penetration Enhancers," 2nd ed., ed. by Smith E. W., Maibach H. I., CRC, Boca Raton, 2006, pp. 3-15.

17) Pappinen S., Urtti A., "Percutaneous Penetration Enhancers," 2nd ed., ed. by Smith E. W., Maibach H. I., CRC, Boca Raton, 2006, pp. 109116.

18) Delgado-Charro M. B., Iglesias-Vilas G., Blanco-Mendez J., LopezQuintela M. A., Marty J. P., Guy R. H., Eur. J. Pharm. Biopharm., 43, 37-42 (1997).

19) Gao S., Sing J., Int. J. Pharmaceut., 165, 45-55 (1998).

20) Debacq-Chainiaux F., Borlon C., De Hertogh B., Remacle J., Morvan P.-Y., Vallee R., Toussaint O., J. Pharm. Pharmacol., 58, 1577-1583 (2006).

21) Lin J.-Y., Selim M. A., Shea C. R., Grichnik J. M., Omar M. M., Monteiro-Riviere N. A., Pinnell S. R., J. Am. Acad. Dermatol., 48, 866874 (2003).

22) Fujii M., Yamanouchi S., Hori N., Iwanaga N., Kawaguchi N., Matsumoto M., Biol. Pharm. Bull., 20, 249-254 (1997).

23) Lee P. J., Langer R., Shastri V. P., Pharm. Res., 20, 264-269 (2003). 